\title{
A redução vocabular como processo de criação lexical: uma análise do fenômeno na Libras
}

\author{
Vocabulary reduction as a lexical creation process: an \\ analysis of this phenomenon in Libras
}

\section{Reducción vocabular como proceso de creación lexical: un análisis del fenómeno en Libras}

\author{
(iD) Delmir Rildo Alves \\ Universidade Federal de Juiz de Fora (UFJF), Juiz de Fora, MG - Brasil \\ delmir10@yahoo.com.br \\ ID Aline Garcia Rodero-Takahira \\ Universidade Federal de Juiz de Fora (UFJF), Juiz de Fora, MG - Brasil \\ rodero.takahira@ufjf.edu.br
}

\begin{abstract}
Resumo: A redução vocabular considera casos em que um composto é encurtado. Dados da Libras sugerem que esse fenômeno ocorre nessa língua. Nossa pesquisa tem como objetivo investigar a mudança em compostos da Libras. Propomos: (a) revisar a bibliografia; (b) formar um corpus considerando sinais presentes em Oates (1969 e 2017); (c) analisar sinais compostos da Libras que sofreram redução, verificando o tipo de apagamento e se há sistematicidade. Analisamos redução vocabular como um processo de formação sinais na Libras proveniente de um corte morfológico que ocorre em todos os tipos de compostos e está intimamente ligado à economia linguística.
\end{abstract}

Palavras-chave: Criação Lexical. Redução Vocabular. Economia Linguística. Libras.

Abstract: Vocabulary reduction considers cases in which a compound is reduced. Data from Libras suggest that the same phenomenon occurs in this language. Our research aims at investigating the change 
in the realization of compounds in Libras. We propose: (a) reviewing bibliography; (b) forming a corpus considering signs present in Oates (1969 and (2017); (c) analising compounds in Libras that went trhough reduction, verifying the kind of deletion and whether there is systematicity. We analyse vocabular reduction as a word formation proccess in Libras that occurs through a morphological cut that can happen in all kinds of compounds and that is deeply linked to linguistics economy.

Keywords: Lexical Creation. Vocabulary Reduction. Linguistics Economy. Libras.

Resumen: La reducción de vocabulario considera los casos en los cuales se acorta un compuesto. Datos sobre la Libras sugieren que ocorre este fenómeno en ese idioma. Nuestra investigación tiene el objetivo de estudiar el cambio en los compuestos de Libras. Proponemos: (a) revisar la bibliografía; (b) formar un corpus considerando señales presentes en Oates (1969 y 2017); (c) analizar las señales compuestas de la Libras que sufrieron reducciones, verificando los tipos de supresiones y si estos ocurrieron de manera sistemática. Analizaremos la reducción de vocablos como un proceso de formación de señales en la Libras mediante un corte morfológico que ocurre en todo tipo de compuestos y que está íntimamente relacionado a la economía lingüística.

Palabras clave: Creación léxica. Reducción de Vocabulario. Economía Lingüística. Libras.

Submetido em 15 de julho de 2020. Aceito em 15 de dezembro de 2020. Publicado em 20 de julho de 2021. 
A redução vocabular como processo de criação lexical: uma análise do fenômeno na LibrasLuiz Delmir Rildo Alves • Aline Garcia Rodero-Takahira

\section{Introdução}

O processo de formação de palavras em uma língua pode se dar de diferentes formas por: derivação prefixal - processo no qual é acrescentado um afixo na anteposição da palavra primitiva acrescentando-lhe uma nova informação, exemplo: infeliz; derivação sufixal - processo no qual é acrescido um afixo ao final do radical agregando um novo sentido, exemplo: carrinho; composição - processo no quais dois morfemas livres se justapõem ou se aglutinam para formarem uma nova palavra, exemplo: guarda-chuva; dentre outros (CUNHA e CINTRA, 2013).

Alguns processos de formação de palavras por serem considerados não-concatenativos são deixados à margem, entre eles estão fenômenos como o truncamento e a redução vocabular. Esses são considerados processos menos produtivos, mas não menos importantes, que contribuem para a expansão lexical (ALVES, 1990, p. 68). O truncamento ${ }^{1}$ é visto como um processo morfofonológico em que ocorre a supressão de uma parte da palavra e também pode ocorrer o acréscimo de uma vogal temática, exemplos: faculdade $>$ facu, neurose $>$ neura, botequim $>$ boteco (VILELA et al., 2006). Já a redução vocabular em tela é tratada como um processo em que ocorre um corte morfológico em palavras compostas como, por exemplo, fotografia > foto (VILELA et al., 2006).

Sobre a redução vocabular, podemos afirmar que não está presente apenas na língua portuguesa, pois trata-se de uma expressão do fenômeno da economia linguística, fenômeno basilar das línguas naturais (MARTINET, 1964). Logo, a redução vocabular ocorre em várias línguas, como na língua portuguesa (otorrinolaringologista > otorrino, odontologia > odonto), na inglesa (telephone $>$ phone, photograph $>$ photo) e na espanhola (autobús $>$ bus, kilogramo $>$ kilo) $^{2}$. Dados da Língua Brasileira de Sinais (Libras) como "neve", realizado pelos sinais BRANCO $>$ NEVE e, posterior-

1 Veja Scher (2012 e 2017) para um tratamento morfossintático das formas truncadas do português brasileiro.

$2 \mathrm{Na}$ língua inglesa e na língua espanhola encontramos este fenômeno com os nomes de "clipping" e "acortamiento", respectivamente, e não parecem se diferenciar do truncamento. 
A redução vocabular como processo de criação lexical: uma análise do fenômeno na LibrasLuiz Delmir Rildo Alves • Aline Garcia Rodero-Takahira

mente, realizado apenas pelo sinal NEVE, sugerem que o mesmo fenômeno ocorre nessa língua.

Pesquisas já realizadas em relação às línguas de sinais comprovam que alguns compostos passaram por um processo de deletação (o que vimos chamando de redução vocabular) e, assim, se tornaram sinais unitários, conforme Frishberg (1975), na ASL (Língua de Sinais Americana, do inglês, American Sign Language), e Diniz (2010), na Libras.

A partir dos apontamentos feitos até aqui, algumas questões de pesquisa se colocam:

1. Na Libras também surgem novas palavras a partir de um corte morfológico, isto é, por meio da redução vocabular? Este fenômeno é produtivo?

2. Com o corte morfológico da palavra-matriz, de que lado se mantém a base na Libras? Existe uma regularidade?

Nossa pesquisa tem como objetivo investigar como se dá o processo de criação de palavras por meio da redução vocabular na Libras, descrevendo e analisando a mudança na sinalização de compostos da Libras ao longo das últimas décadas. Para tanto, nos apoiamos na análise de Vilela et al. (2006), que considera a redução vocabular como um processo morfológico e não morfofonológico e que ocorre apenas em compostos, diferenciando-se assim do truncamento. Já os objetivos específicos são:

a) montar um corpus para análise formado por um levantamento de dados de sinais compostos na Libras em registros antigos e encontrar os mesmos sinais em materiais mais recentes, compreendendo um bom espaço de tempo para visualizar possíveis mudanças;

b) analisar os possíveis sinais compostos ${ }^{3}$ da Libras que sofreram um corte morfológico, verificando a tendência de apagamen-

\footnotetext{
3 Utilizamos a expressão "possíveis compostos" para sinais formados por meio de dois ou mais sinais que realizados sequencialmente levam a um significado diferente, no entanto, devido ao caráter de nosso corpus não analisamos a formação destes sinais por meio das regras morfológicas e fonológicas, como aquelas apresentadas por Klima e Bellugi (1979) e Liddell e Johnson (1986), sobre a ASL, e em Quadros e Karnopp (2004) e Rodero-Takahira (2015), sobre a Libras.
} 
A redução vocabular como processo de criação lexical: uma análise do fenômeno na LibrasLuiz Delmir Rildo Alves • Aline Garcia Rodero-Takahira

to/manutenção de uma das bases (à direita ou à esquerda) e a sistematicidade, ou não, do fenômeno.

Para alcançar esses objetivos, realizamos o estudo bibliográfico que aborda o processo de redução vocabular na língua portuguesa e de deletação em sinais na Libras e na ASL (American Sign Language). Em seguida, partimos para a catalogação dos sinais compostos presentes no livro Linguagem das Mãos (1969), de Eugênio Oates, e para uma análise comparativa com sua versão recente Língua das Mãos (2017), com adaptação e atualização de Simone Vecchio, visando coletar os possíveis compostos. Uma vez tendo nosso corpus organizado, passamos a observar se houve, ou não, mudanças no registro das sinalizações dentro desse intervalo de quase 50 anos. Feitas essas etapas, realizamos uma análise dos sinais que apresentaram mudanças e percebemos que muitos deles sofreram redução vocabular, ou seja, apresentaram um corte morfológico com o apagamento de uma de suas bases, e tecemos, então, algumas considerações a respeito desse fenômeno e dos dados analisados.

Por fim, ressaltamos que este trabalho visa contribuir com as pesquisas que envolvem os processos de criação lexical na Libras. Analisamos a redução vocabular como um processo de formação sinais na Libras proveniente de um corte morfológico que pode ocorrer tanto em compostos sequenciais quanto em compostos simultâneos ou simultâneo-sequenciais. Enfatizamos que este processo é comum nas línguas naturais, pois o mesmo está intimamente ligado à economia linguística.

\section{O fenômeno da redução vocabular e a economia linguística nas línguas naturais}

A redução vocabular é um processo de formação de palavras em que o item lexical sofre uma redução, no entanto, mantém o significado da palavra-matriz. Conforme os estudos de Vilela et al. (2006), os fenômenos nos quais ocorrem a redução na língua 
A redução vocabular como processo de criação lexical: uma análise do fenômeno na LibrasLuiz Delmir Rildo Alves • Aline Garcia Rodero-Takahira

portuguesa são abordados na maioria das gramáticas e nos manuais de morfologia como truncamento, redução vocabular, abreviação e siglagem, não tendo uma distinção clara entre eles. As autoras, com base nas pesquisas feitas por Araújo (2002) e Gonçalves (1999), propõem em suas análises uma diferenciação entre truncamento e redução vocabular. A redução vocabular, segundo as pesquisadoras, é um fenômeno essencialmente morfológico e acontece exclusivamente em compostos. Para elas, "a redução é um corte morfológico, que desfaz a composição que deu origem à palavra-matriz" (VILELA et al., 2006). Como exemplos de redução vocabular as autoras citam: fotografia (foto+grafia) > foto; retro-projetor (retro+projetor) > retro; lipoaspiração (lipo+aspiração) > lipo. Considerando os dados analisados, as autoras afirmam que há uma tendência de se manterem as bases esquerdas dos compostos. Já o truncamento seria um processo morfofonológico em que, além da supressão de uma parte da palavra, também pode ocorrer o acréscimo de uma vogal temática. Como exemplos de truncamento, temos: faculdade $>$ facu, neurose $>$ neura, botequim> boteco.

As autoras também diferenciam o truncamento da redução vocabular pelo viés semântico-pragmático. Elas consideram que o truncamento é usado em expressões pejorativas, jocosas ou de cunho afetivo. Já a redução estaria apenas expressando intimidade em relação ao tema na qual a palavra é reduzida. Portanto, o truncamento possui traço de gíria. Já a redução parece não ter tal valor, podendo ser usada por vários grupos sociais (VILELA et al., 2006).

Além do exposto, consideramos a redução vocabular como uma expressão do fenômeno da economia linguística. Em relação a este fenômeno, Martinet (1964) relata que: “A evolução linguística pode considerar-se regida pela antinomia permanente entre as necessidades comunicativas do homem e a sua tendência para reduzir ao mínimo a actividade mental e física". Ainda segundo Martinet (1964), a economia linguística atua nos eixos paradigmáticos e sintagmáticos da língua, sendo considerada a forma motriz das mudanças formais e semânticas. 
A redução vocabular como processo de criação lexical: uma análise do fenômeno na LibrasLuiz Delmir Rildo Alves • Aline Garcia Rodero-Takahira

Bagno (2011) afirma que a economia linguística engloba processos que representam mecanismos de mudança que buscam reagir positivamente aos seguintes impulsos: "poupar a memória, o processamento mental e a realização física da língua, eliminando os aspectos redundantes e as articulações mais exigentes" e "preencher lacunas na gramática da língua, de modo a torná-la mais eficiente como instrumento de interação sociocomunicativa". Sendo assim, o fenômeno da economia linguística é um fenômeno basilar das línguas naturais, portanto, este fenômeno se expressa em várias línguas, se não em todas elas.

\section{A criação lexical nas línguas de sinais via compostos e o fenômeno da redução vocabular}

Conforme exposto na sessão anterior, consideramos que a redução vocabular ocorre apenas em compostos. Portanto, antes de verificarmos se tal fenômeno ocorre frequentemente na Libras, abordaremos brevemente o processo de composição.

A composição é um fenômeno linguístico de formação de palavras no qual se utiliza de itens lexicais, que são morfemas livres, que se justapõem ou se aglutinam para formarem um novo item lexical (FELIPE, 2006).

O fenômeno da composição parece ser muito produtivo nas línguas de sinais. Muitos pesquisadores vêm estudando este processo de criação lexical, entre eles, Klima e Bellugi (1979) e Liddell e Johnson (1986), para a ASL, e Quadros e Karnopp (2004), Felipe (2006), Figueiredo Silva e Sell (2009), Rodero-Takahira (2015) e Rodero-Takahira e Scher (2020), para a Libras. Na LGP (Língua de Sinais Portuguesa, do português de Portugal, Língua Gestual Portuguesa), Mineiro et al. (2007, apud FARIA-NASCIMENTO \& CORREIA, 2011) afirmam que: 
A redução vocabular como processo de criação lexical: uma análise do fenômeno na LibrasLuiz Delmir Rildo Alves • Aline Garcia Rodero-Takahira

“[...] a composição de gestos parece ser uma alternativa representativa. Tal facto pode dever-se a um aproveitamento do material linguístico disponível (gestos existentes) para a criação de novas denominações. Assim se manifesta a capacidade da LGP para recrutar processos morfológicos econômicos. Tal como as outras línguas de modalidades orais ou gestuais, a LGP possui um conjunto de regras que lhe permite gerar uma quantidade de enunciados e gestos infinita".

Na Libras, encontramos diversos compostos como, por exemplo, o sinal de "medicina", que é formado pelo sinal de MÉDICO seguido do sinal de ESTUDAR.

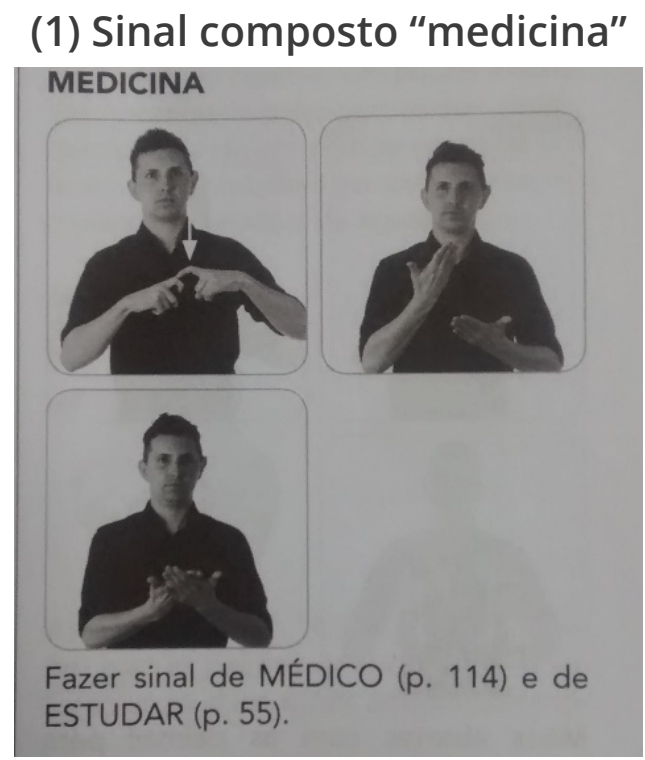

(OATES, 2017, p. 113)

Rodero-Takahira (2015) e Rodero-Takahira e Scher (2020) trazem uma série de exemplos de sinais compostos na Libras, classificando-os em compostos sequenciais, como "escola" (CASA>ESTU$\mathrm{DO}^{4}$ ); simultâneos, como bichinho de balanço (sinal classificador para ANIMAL >> sinal classificador para PESSOA5); e, simultâneos-

4 Onde ">" indica a sequencialidade na sinalização.

5 Onde ">>" indica a simultaneidade na sinalização. O sinal classificador para ANIMAL é realizado com a CM em "5" com os dedos unidos e o sinal classificador para PESSOA é realizado com a CM em "2" com os dedos distendidos. 
A redução vocabular como processo de criação lexical: uma análise do fenômeno na LibrasLuiz Delmir Rildo Alves • Aline Garcia Rodero-Takahira

-sequenciais, como "cavalinho de balanço" (CAVALO > sinal classificador para ANIMAL >> sinal classificador para PESSOA).

A pesquisadora Frishberg (1975), ao realizar um estudo pioneiro sobre o processo de mudança histórica na ASL, percebeu que, entre as mudanças, alguns sinais compostos passaram a ser sinais simples. A pesquisadora também verificou por meio das mudanças dos sinais um movimento da iconicidade para a arbitrariedade. Ela cita como exemplo o sinal FEEL (sentimento), que era realizado no lado esquerdo do peito, fazendo referência à localização do coração, que por milênios foi associado aos sentimentos e que passa a ser realizado no centro do peito, perdendo assim a iconicidade presente no ponto de articulação.

Como exemplo de sinal composto que sofre deletação, Frishberg (1975) cita o sinal de "pássaro":

(2) Sinal composto "pássaro"

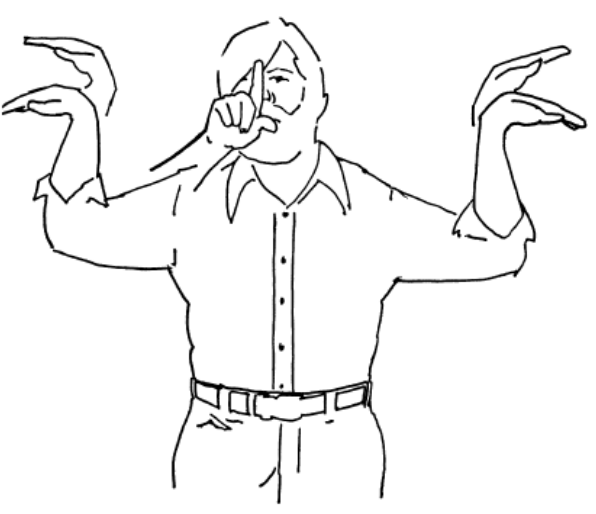

(a)

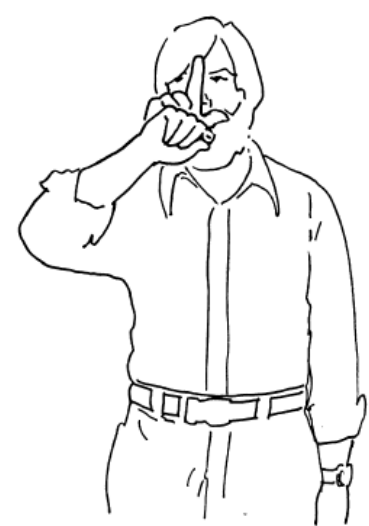

(b)

(FRISHBERG, 1975, p. 709)

O sinal de "pássaro", no primeiro momento, forma antiga em (a) é realizado com uma mão em frente ao rosto para a realização do sinal classificador (CL) de "BICO" e em seguida pelas duas mãos para o sinal CL de "ASAS". No segundo momento, forma moderna em (b), a segunda parte do sinal composto é deletada.

Em relação à Libras, um dos primeiros estudos que abordam as mudanças históricas é o trabalho de Diniz (2010). Assim como 
A redução vocabular como processo de criação lexical: uma análise do fenômeno na LibrasLuiz Delmir Rildo Alves • Aline Garcia Rodero-Takahira

Frishberg (1975), Diniz (2010), em suas análises também encontrou sinais compostos que passaram a ser simples, como os sinais de "pimenta" e "confessar".

(3) Sinal "pimenta"

\begin{tabular}{|l|l|l|l|}
\hline GLOSA & ICON. & OATES & INES \\
\hline Est. 2 & PIMENTA & & \\
& & & \\
& & & \\
& &
\end{tabular}

(DINIZ, 2010, p. 93)

Como podemos verificar, o fenômeno da deletação abordado por Frishberg (1975) e Diniz (2010) para a língua de sinais é o mesmo fenômeno abordado por Vilela et al. (2006) como redução vocabular em relação ao Português Brasileiro (PB); uma vez que os exemplos, citados pelas pesquisadoras das línguas de sinais, também são compostos porque sofreram um corte justamente no limite morfológico e que mantêm o significado do sinal-matriz.

É interessante observar que o fenômeno da economia linguística, abordado acima, se expressa nas línguas de sinais tanto para criação lexical, via compostos (usando sinais já existentes na língua para criar outros sinais de forma econômica), quanto no processo de criação lexical, via redução vocabular (deixando o processo ainda mais econômico). 
A redução vocabular como processo de criação lexical: uma análise do fenômeno na LibrasLuiz Delmir Rildo Alves • Aline Garcia Rodero-Takahira

\section{Metodologia de organização do corpus}

Como mencionado anteriormente, neste trabalho nosso foco é verificar se o fenômeno da redução vocabular é frequente na Libras e se há uma preferência por manutenção de uma das bases (à direita ou à esquerda). Para tal investigação, precisamos, inicialmente, formar um corpus bastante abrangente em relação temporal para que possamos observar as possíveis mudanças em compostos e se o processo de redução é frequente na Libras ou não.

Para a visualização dos dados, o primeiro material escolhido foi o livro Linguagem das Mãos, de Oates (1969). Eugênio Oates foi um padre redentorista norte-americano que veio ao Brasil como missionário. O livro é fruto de um trabalho que durou anos e contou com a colaboração do Pe. Penido Bunier ${ }^{6}$. O livro apresenta um número significativo de sinais e teve grande repercussão no Brasil durante várias décadas. Este livro é a primeira publicação do século XX de que se tem notícia na qual consta um glossário da Libras.

Figura 1 - Capa do livro Linguagem das Mãos (OATES, 1969)

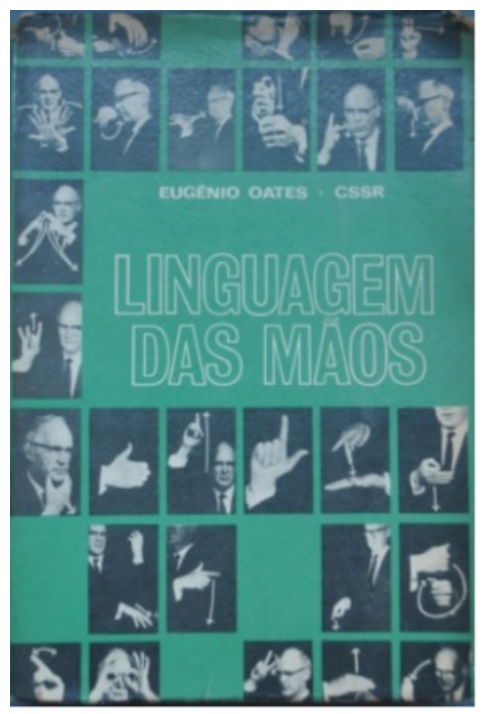

Fonte: Oates (1969)

$\overline{60}$ Padre Vicente de Paulo Penido Burnier é natural de Juiz de Fora, Minas Gerais. É considerado o primeiro padre surdo do Brasil e teve uma participação bastante ativa nas associações dos surdos. 
A redução vocabular como processo de criação lexical: uma análise do fenômeno na LibrasLuiz Delmir Rildo Alves • Aline Garcia Rodero-Takahira

O segundo material analisado é o livro Língua das Mãos (2017), que é uma versão atualizada e adaptada por Simone Vecchio do livro Linguagem das Mãos. A nova edição, além de atualizar alguns vocábulos, acrescenta novos sinais, como, por exemplo, os sinais relacionados às mídias sociais. Também a estrutura do livro foi mudada com o intuito de facilitar a localização dos termos.

Figura 2 - Capa do livro Língua das Mãos (OATES, 2017)

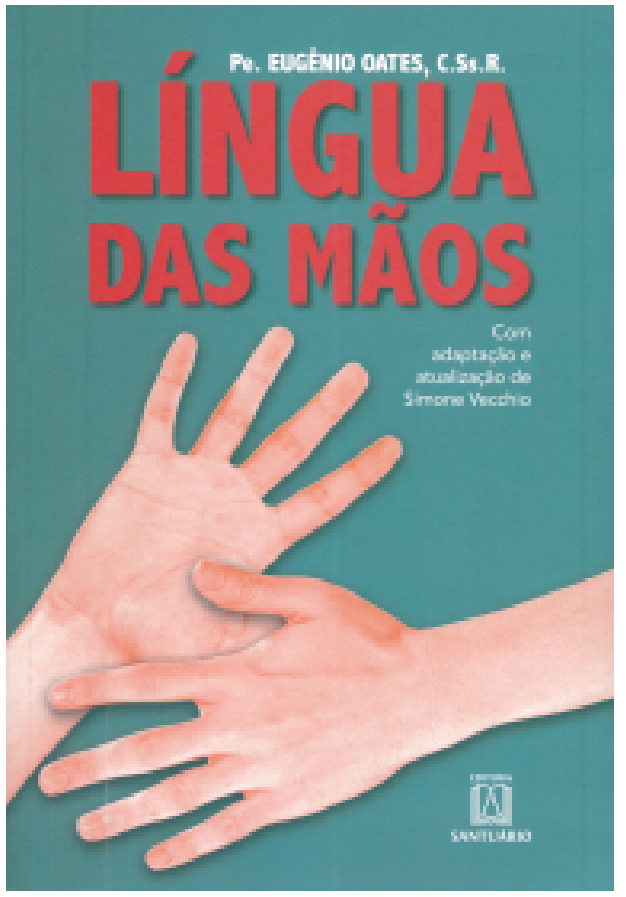

Fonte: Oates (2017)

Como procedimento, catalogamos todos os possíveis compostos presentes em Oates (1969). Utilizamos esse livro como ponto de partida por se tratar do mais antigo e com republicação atualizada bastante recente, o que nos dá um intervalo de quase 50 anos para comparação da evolução dos sinais. Em seguida, comparamos os vocábulos encontrados com os mesmos vocábulos apresentados na versão atualizada, Oates (2017).

A seguir apresentamos o quadro inicial com os 41 possíveis compostos que selecionamos em Oates (1969) e Oates (2017). As glosas apresentadas no quadro foram feitas por nós a partir das 
A redução vocabular como processo de criação lexical: uma análise do fenômeno na LibrasLuiz Delmir Rildo Alves • Aline Garcia Rodero-Takahira

descrições e explicações ou das fotos dos sinais apresentados nas obras e com base na forma de anotação de compostos apresentada em Rodero-Takahira (2015) e Rodero-Takahira e Medeiros (manuscrito) ${ }^{7}$.

\section{Quadro 1 - Sinais compostos e suas respectivas formas reduzidas} encontrados em Oates (1969 e 2017)

\begin{tabular}{|c|c|c|c|}
\hline \multicolumn{2}{|c|}{ Entrada } & \multirow{2}{*}{$\begin{array}{l}\begin{array}{l}\text { Realização do sinal em Oates } \\
\text { (1969) }\end{array} \\
\text { BENÇÃO>TRAÇADO-DE-CRUZ }\end{array}$} & \multirow{2}{*}{$\begin{array}{l}\begin{array}{l}\text { Realização do sinal em } \\
\text { Oates (2017) }\end{array} \\
\text { BENÇÃO }\end{array}$} \\
\hline 1) & Abençoar & & \\
\hline 2) & Achar & $\begin{array}{l}\text { VER>ENTIDADE-RETA-APROXI- } \\
\text { MAR>> ENTIDADE-RETA-APROXIMAR }\end{array}$ & $\begin{array}{l}\text { ENTIDADE-RETA-APROXI- } \\
\text { MAR > > ENTIDADE-RETA- } \\
\text {-APROXIMAR }\end{array}$ \\
\hline 3) & Camisa & SEGURAR-CAMISA>HOMEM & SEGURAR-CAMISA \\
\hline 4) & Canário & BICO>AMARELO>CANTAR & BICO>AMARELO \\
\hline 5) & Casar & COLOCAR-ANEL>>MÃO>CASAD@ & CASAD@ \\
\hline 6) & Cobra & $\begin{array}{l}\text { LIINGUA-BIFURCADA-MOVER>ENTI- } \\
\text { DADE-RETA-ANDAR }\end{array}$ & $\begin{array}{l}\text { LÍNGUA-BIFURCADA-MO- } \\
\text { VER }\end{array}$ \\
\hline 7) & Coco & $\begin{array}{l}\text { ENTIDADE-REDONDA-SACUDIR>EN- } \\
\text { TIDADE-REDONDA>>CORTAR-COM- } \\
\text {-FACA }\end{array}$ & $\begin{array}{l}\text { ENTIDADE-REDONDA-SA- } \\
\text { CUDIR }\end{array}$ \\
\hline 8) & Cola & $\begin{array}{l}\text { DEDOS-COLAR }>\text { MÃO-PRESSIO- } \\
\text { NAR }>>\text { ENTIDADE-PLANA }\end{array}$ & DEDOS-COLAR \\
\hline 9) & Cuidado & VER $>$ CAUTELA & CAUTELA \\
\hline 10) & Elevador & $\begin{array}{l}\text { APERTAR-BOTÃO>ELEVADOR-SU- } \\
\text { BIR>>PAREDE-ELEVADOR }\end{array}$ & $\begin{array}{l}\text { ELEVADOR-SUBIR>>PARE- } \\
\text { DE-ELEVADOR }\end{array}$ \\
\hline 11) & Farmácia & $\begin{array}{l}\text { CASA>MANIPULAR-REMÉDIO>>RECI- } \\
\text { PIENTE }\end{array}$ & $\begin{array}{l}\text { M A N I P ULAR - R E M É - } \\
\text { DIO>>RECIPIENTE }\end{array}$ \\
\hline 12) & Feira & FEIRA>SEGURAR-SACOLA & FEIRA \\
\hline 13) & Foguete & $\begin{array}{l}\text { RISCAR >>ACENDEDOR>SEGURAR- } \\
\text {-FOGUETE }\end{array}$ & SEGURAR-FOGUETE \\
\hline 14) & Formatura & ESTUDAR>PEGAR-CANUDO & PEGAR-CANUDO \\
\hline 15) & Futebol & FUTEBOL>PÉ-CHUTAR>>ENTIDADE & FUTEBOL \\
\hline 16) & Giz & $\begin{array}{l}\text { ESCREVER-LOUSA>BRANCO> TAMA- } \\
\text { NHO-DO-GIZ }\end{array}$ & ESCREVER-LOUSA \\
\hline 17) & Gripe & FEBRE>PASSAR-LENÇO-NARIZ & PASSAR-LENÇO-NO-NARIZ \\
\hline
\end{tabular}

\footnotetext{
$7 \mathrm{Na}$ coluna onde detalhamos os sinais compostos encontrados em Oates (1969), detalhamos a realização de compostos sequenciais, simultâneos e simultâneo-sequenciais utilizando ">" para indicar a sequencialidade e " $>$ " para indicar a simultaneidade na sinalização. Optamos por não detalhar morfemas classificadores na sinalização dos compostos que selecionamos, uma vez que não entraremos na discussão desse tipo de dados neste trabalho. Para mais informações sobre os três tipos de compostos na Libras, veja Rodero-Takahira (2015) e Rodero-Takahira e Scher (2020).
} 
A redução vocabular como processo de criação lexical: uma análise do fenômeno na LibrasLuiz Delmir Rildo Alves • Aline Garcia Rodero-Takahira

\begin{tabular}{|c|c|c|c|}
\hline 18) & Hospital & CASA>TRAÇADO-DE-CRUZ-NA-TESTA & $\begin{array}{l}\text { TRAÇAD O-DE-CRUZ-NA- } \\
\text {-TESTA }\end{array}$ \\
\hline 19) & Inquérito & ADVOGADO>PERGUNTAR & PERGUNTAR \\
\hline 20) & Jordânia & TRAÇADO-DO-TURBANTE>ARÁBIA & TRAÇADO-DO-TURBANTE \\
\hline 21) & Juiz & JUDICIAL>BATER-MATELO & JUDICIAL \\
\hline 22) & Juiz Esportivo & $\begin{array}{l}\text { APITO-SOPRAR>>SEGURAR-BANDEI- } \\
\text { RINHA-ACENAR }\end{array}$ & APITO-SOPRAR \\
\hline 23) & Lenço & $\begin{array}{l}\text { TIRAR-LENÇO-DO-BOLSO>PASSAR- } \\
\text {-LENÇO-NO-NARIZ }\end{array}$ & PASSAR-LENÇO-NO-NARIZ \\
\hline 24) & Mala & $\begin{array}{l}\text { FORMA-RETANGULAR>SEGURAR- } \\
\text {-MALA }\end{array}$ & SEGURAR-MALA \\
\hline 25) & México & $\begin{array}{l}\text { TRAÇADO-DA-ABA-DO-SOMBREIRO- } \\
\text {-MEXICANO>TOCAR-GUITARRA }\end{array}$ & $\begin{array}{l}\text { TRAÇADO-DA-ABA-DO- } \\
\text {-SOMBREIRO-MEXICANO }\end{array}$ \\
\hline 26) & Neve & BRANCO>NEVE & NEVE \\
\hline 27) & Óculos & $\begin{array}{l}\text { ENTIDADE-CIRCULAR>TRAÇADO- } \\
\text {-DAS-ALÇAS- DOS-ÓCULOS }\end{array}$ & ENTIDADE-CIRCULAR \\
\hline 28) & Onda & ÁGUA>TRAÇADO-DAS-ONDAS & TRAÇADO-DAS-ONDAS \\
\hline 29) & Pimenta & $\begin{array}{l}\text { TOCAR-DEDO-NA-LÍNGUA >ABANAR- } \\
\text {-BOCA }\end{array}$ & ABANAR-BOCA \\
\hline 30) & Prender & AGARRAR>GRADE & GRADE \\
\hline 31) & Prometer & FALAR>PROMESSA & PROMESSA \\
\hline 32) & Protestante & LIVRO-ABERTO>MEDITAR & LIVRO-ABERTO \\
\hline 33) & Régua & $\begin{array}{l}\text { MADEIRA>TRAÇAR-LINHA>>ENTIDA- } \\
\text { DE-RETA }\end{array}$ & $\begin{array}{l}\text { TRAÇAR-LINHA>> ENTIDA- } \\
\text { DE-RETA }\end{array}$ \\
\hline 34) & Roupa & SEGURAR-CAMISA>ALISAR-CAMISA & SEGURAR-CAMISA \\
\hline 35) & Serra & $\begin{array}{l}\text { TAMANHO-DA-SERRA>ENTIDADE>>- } \\
\text { SEGURAR-SERRA-SERRAR }\end{array}$ & $\begin{array}{l}\text { ENTIDADE>>SEGURAR-SER- } \\
\text { RA-SERRAR }\end{array}$ \\
\hline 36) & Serrote & $\begin{array}{l}\text { TAMANHO-DO-SERROTE> ENTIDA- } \\
\text { DE }>>\text { SEGURAR-SERRA-SERRAR }\end{array}$ & $\begin{array}{l}\text { ENTIDADE>>SEGURAR-SER- } \\
\text { RA-SERRAR }\end{array}$ \\
\hline 37) & Tempo & $\begin{array}{l}\text { TEMPO>BOM ou TEMPO>RUIM ou } \\
\text { TEMPO>CHUVA }\end{array}$ & TEMPO \\
\hline 38) & Tênis & $\begin{array}{l}\text { SEGURAR-RAQUETE>>SEGURAR-BO- } \\
\text { LA-JOGAR>SEGURAR-RAQUETE-BA- } \\
\text { TER }\end{array}$ & SEGURAR-RAQUETE-BATER \\
\hline 39) & Tubarão & $\begin{array}{l}\text { PEIXE-NADAR>SUPERFICE-DE-Á- } \\
\text { GUA>>BARBATANA-MOVER }\end{array}$ & $\begin{array}{l}\text { S U P E R F I C E - D E - Á - } \\
\text { GUA >>BARBATANA-MOVER }\end{array}$ \\
\hline 40) & Voar & ASAS-MOVER>>PÁSSARO-VOAR & ASAS-MOVER \\
\hline 41) & Xícara & $\begin{array}{l}\text { ENTIDADE-CIRCULAR>PIRES>>SEGU- } \\
\text { RAR-ASA-DA-XÍCARA-BEBER }\end{array}$ & $\begin{array}{l}\text { PIRES>>SEGURAR-ASA-DA- } \\
\text {-XÍCARA-BEBER }\end{array}$ \\
\hline
\end{tabular}

Fonte: elaboração dos autores (2020).

Na próxima seção trazemos a análise e discussão sobre os dados observados acima. 
A redução vocabular como processo de criação lexical: uma análise do fenômeno na LibrasLuiz Delmir Rildo Alves • Aline Garcia Rodero-Takahira

\section{Redução vocabular na Libras}

Em Oates (1969) foram encontrados 353 sinais que foram considerados por nós como possíveis compostos, inicialmente, por serem formados por dois ou três sinais e levarem a um significado diferente das partes que os compõem. Na análise comparativa com a versão atualizada do livro, Oates (2017), encontramos 41 sinais $^{8}$ que sofreram algum tipo de apagamento que, posteriormente, analisamos e vimos se tratar de um corte morfológico com apagamento de uma das bases do composto, configurando assim a redução vocabular.

Dois exemplos de redução vocabular encontrados na pesquisa são os sinais de "canário" e de "farmácia". O sinal de "canário" em Oates (1969) é formado pelos sinais BICO>AMARELO>CANTAR. Já na versão atualizada do livro, encontra-se BICO>AMARELO.

(4) Sinal para "canário"9

\begin{tabular}{|c|l|l|}
\hline GLOSA & OATES (1969) \\
\hline CANÁRIO & $\begin{array}{l}\text { CANARIO - (864) - Indicar o bico } \\
\text { dêste pássaro, colocando a mão di- } \\
\text { reita diante da bôca, unindo e se- } \\
\text { parando o polegar e indicador, os } \\
\text { demais dedos fechados. Em segui- } \\
\text { da, fazer a mímica de "amarelo" } \\
\text { a de "cantar". }\end{array}$ \\
\hline
\end{tabular}

(OATES, 1969, p. 201 e OATES, 2017, p. 165)

Podemos observar que, nesse exemplo, após o corte morfológico manteve-se as bases esquerdas BICO>AMARELO.

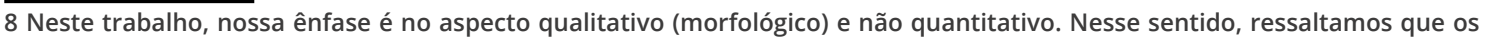
números dos sinais que sofreram a redução vocabular podem variar, conforme a obra a ser comparada, devido à variação linguística.

9 Observamos que em Oates (1969) o termo "mímica" é usado. Vale ressaltar que na época eram poucos os estudos linguísticos que consideravam as línguas de sinais enquanto línguas naturais, tendo essa visão formalmente se iniciado na própria década de 1960 com a publicação de Stokoe (1960) sobre a formação dos sinais em ASL. Onde lê-se "mímica" nos recortes da obra, entendemos que são sinais, ou palavras da Libras. 
A redução vocabular como processo de criação lexical: uma análise do fenômeno na LibrasLuiz Delmir Rildo Alves • Aline Garcia Rodero-Takahira

Já o sinal de "farmácia", antes formado pelos sinais de CASA>MANIPULAR-REMÉDIO>>RECIPIENTE, foi encontrado como MANIPULAR-REMÉDIO>>RECIPIENTE.

(5) Sinal para "farmácia"

\begin{tabular}{|c|c|c|}
\hline GLOSA & OATES (1969) & OATES (2017) \\
\hline FARMÁCIA & &
\end{tabular}

(OATES, 1969, p. 116 e OATES, 2017, p. 103)

Nesse sinal, após o corte morfológico, a base direita REMÉDIO permaneceu.

Em nossa análise, verificamos que a redução vocabular ocorre em diferentes classes gramaticais e tanto do lado esquerdo quanto do lado direito. Em relação aos verbos, encontramos "abençoar" e "voar" que sofreram o corte morfológico e mantiveram a base esquerda.

(6) Sinal para "abençoar"

\begin{tabular}{|c|c|c|}
\hline GLOSA & OATES (1969) & OATES (2017) \\
\hline ABENÇOAR & (1) \\
\hline
\end{tabular}

(OATES, 1969, p. 17 e OATES, 2017, p. 31) 
A redução vocabular como processo de criação lexical: uma análise do fenômeno na LibrasLuiz Delmir Rildo Alves • Aline Garcia Rodero-Takahira

Já os verbos "achar", "casar", "prender" e "prometer" sofreram o corte morfológico e mantiveram a base direita.

(7) Sinal para "achar (encontrar)"

\begin{tabular}{|c|c|c|c|}
\hline GLOSA & \multicolumn{2}{|c|}{ OATES (1969) } & OATES (2017) \\
\hline ACHAR & ${ }^{7 \mathrm{~A}}$ (Encontrar) & (18 &
\end{tabular}

(OATES, 1969, p. 18 e OATES, 2017, p. 32)

No que concerne aos substantivos, encontramos a redução vocabular presente tanto em substantivos comuns quanto em substantivos próprios. Os substantivos comuns que sofreram o corte morfológico e que mantiveram a base esquerda foram: "camisa", "canário", "cobra", "coco", "cola", "feira", "futebol", "giz", "juiz", "óculos", "protestante", "roupa" e "tempo".

(8) Sinal para "camisa"

\begin{tabular}{|c|c|c|}
\hline GLOSA & OATES (1969) & OATES (2017) \\
\hline CAMISA & & \\
\hline & &
\end{tabular}

(OATES, 1969, p. 274 e OATES, 2017, p. 238)

Com relação aos substantivos comuns que sofreram o corte morfológico e que mantiveram a base direita foram: "cuidado", 
A redução vocabular como processo de criação lexical: uma análise do fenômeno na LibrasLuiz Delmir Rildo Alves • Aline Garcia Rodero-Takahira

"elevador", "farmácia", "formatura", "foguete", "gripe", "hospital", "inquérito", "lenço", "mala", "neve", "onda", “pimenta”, "régua", "serra", "serrote", "tênis", "tubarão" e "xícara".

(9) Sinal para "cuidado"

\begin{tabular}{|c|c|c|}
\hline GLOSA & OATES (1969) & OATES (2017) \\
\hline CUIDADO & (222 A & \\
\hline
\end{tabular}

(OATES, 1969, p. 105 e OATES, 2017, p. 97)

Em relação aos substantivos próprios, encontramos os sinais de "Jordânia" e "México", ambos mantiveram a base esquerda.

(10) Sinal para "Jordânia"

\begin{tabular}{|c|c|c|}
\hline GLOSA & OATES (1969) & OATES (2017) \\
\hline JORDÂNIA & & \\
& &
\end{tabular}

(OATES, 1969, p. 257 e OATES, 2017, p. 223)

Para melhor visualização, a seguir é apresentada a tabela com o nome dos 41 possíveis compostos que sofreram a deletação e a indicação da base deletada. 
A redução vocabular como processo de criação lexical: uma análise do fenômeno na LibrasLuiz Delmir Rildo Alves • Aline Garcia Rodero-Takahira

Quadro 2 - Deletação de base dos possíveis compostos

\begin{tabular}{|c|c|c|c|}
\hline \multicolumn{2}{|c|}{ Entrada } & \multirow[t]{2}{*}{ Deletação da Base Esquerda } & \multirow{2}{*}{$\frac{\text { Deletação da base direita }}{X}$} \\
\hline 1) & Abençoar & & \\
\hline & Achar & $x$ & \\
\hline 3) & Camisa & & $x$ \\
\hline 4) & Canário & & $x$ \\
\hline 5) & Casar & $x$ & \\
\hline 6) & Cobra & & $x$ \\
\hline & Coco & & $x$ \\
\hline 8) & Cola & & $x$ \\
\hline & Cuidado & $x$ & \\
\hline & Elevador & $x$ & \\
\hline & ) Farmácia & $x$ & \\
\hline & Feira & & $x$ \\
\hline & Foguete & $x$ & \\
\hline & Formatura & $x$ & \\
\hline & Futebol & & $x$ \\
\hline 16) & Giz & & $x$ \\
\hline 17) & Gripe & $x$ & \\
\hline & Hospital & $x$ & \\
\hline 19) & Inquérito & $x$ & \\
\hline 20). & ) Jordânia & & $x$ \\
\hline 21). & Juiz & & $x$ \\
\hline & Juiz Esportivo & - & $\bar{L}$ \\
\hline & Lenço & $x$ & \\
\hline & Mala & $x$ & \\
\hline 25) & México & & $x$ \\
\hline & Neve & $x$ & \\
\hline & Óculos & & $x$ \\
\hline & Onda & $x$ & \\
\hline & Pimenta & $x$ & \\
\hline 30) & Prender & $x$ & \\
\hline & Prometer & $x$ & \\
\hline & Protestante & & $x$ \\
\hline & Régua & $x$ & \\
\hline & Roupa & & $x$ \\
\hline 35) & Serra & $x$ & \\
\hline
\end{tabular}


A redução vocabular como processo de criação lexical: uma análise do fenômeno na LibrasLuiz Delmir Rildo Alves • Aline Garcia Rodero-Takahira

\begin{tabular}{|l|c|c|}
\hline 36) Serrote & $X$ & $X$ \\
\hline 37) Tempo & $X$ & \\
\hline 38) Tênis & $X$ & $X$ \\
\hline 39) Tubarão & & \\
\hline 40) Voar & $X$ & \\
\hline 41) Xícara & & \\
\hline
\end{tabular}

Fonte: elaboração dos autores (2020).

Entre os 41 possíveis compostos, verificamos que em apenas um não é possível analisar a perda de sua base pelo viés linear, isto é, do lado esquerdo ou direito, uma vez que, na Libras também existem compostos simultâneos, como é o caso do sinal de "Juiz Esportivo", registrado em Oates (1969) da seguinte forma: "Fazer a mímica de "apito" com a mão direita e, ao mesmo tempo, sacudir rapidamente a mão esquerda fechada, dando a ideia de acenar a bandeirinha". Este sinal, após o corte morfológico, manteve a base "APITO" conforme vemos na imagem a seguir.

(11) Sinal composto para "juiz esportivo"

\begin{tabular}{|c|l|l|}
\hline GLOSA & OATES (1969) & OATES (2017) \\
\hline JUIZ & 1.186 \\
\hline ESPORTIVO
\end{tabular}

(OATES, 1969, p. 289 e OATES, 2017, p. 250)

Com essas observações iniciais do nosso corpus, percebemos que diferentemente do que vem ocorrendo na língua portuguesa, em que a tendência é manter as bases à esquerda da palavra-matriz (Vilela et al. 2006), na Libras parece ser impossível de se observar uma tendência, pois, dos 41 compostos analisados, 17 manti- 
A redução vocabular como processo de criação lexical: uma análise do fenômeno na LibrasLuiz Delmir Rildo Alves • Aline Garcia Rodero-Takahira

veram a base esquerda, 23 a base direita e em 1 não foi possível se analisar a linearidade da base apagada, uma vez que se trata de um composto simultâneo, uma sinalização simultânea. Assim, depreendemos que o corte morfológico na Libras pode ocorrer em todos os tipos de compostos, em compostos sequenciais, simultâneos e simultâneo-sequenciais. Notadamente, em compostos simultâneos há o apagamento do sinal inteiro realizado com uma das mãos, como vimos em (11). Em compostos simultâneo-sequenciais, como "elevador", o primeiro sinal sequencial inteiro "apertar o botão" foi apagado. Não observamos nenhum composto simultâneo sequencial onde foi apagado um dos sinais da parte simultânea.

\section{Considerações finais}

A Libras, assim como qualquer língua, possui diversos processos de formação lexical. Com esta pesquisa foi possível verificar que um dos processos de formação de sinais se dá pela redução vocabular, que é um processo não-concatenativo no qual, por meio de um corte morfológico, ocorre a perda de um morfema. Em nossa pesquisa bibliográfica verificamos que o fenômeno de deletação, abordado por Frishberg (1975) e Diniz (2010), para a língua de sinais é o mesmo fenômeno abordado por Vilela et al. (2006) como redução vocabular em relação ao Português Brasileiro (PB), uma vez que, os exemplos citados pelas pesquisadoras das línguas de sinais também são compostos que sofreram um corte justamente no limite morfológico e que mantêm o significado do sinal-matriz. Também foi possível verificar que a economia linguística se expressa tanto na composição quanto na redução vocabular, uma vez que, no primeiro fenômeno o processo de formação de sinais se dá por morfemas já existentes na língua e o segundo pela deletação de um morfema.

Com o corpus, que foi constituído por 41 sinais que sofreram a redução vocabular, verificou-se que 17 mantiveram a base es- 
A redução vocabular como processo de criação lexical: uma análise do fenômeno na LibrasLuiz Delmir Rildo Alves • Aline Garcia Rodero-Takahira

querda, 23 a base direita e 1 composto simultâneo não permitiu a observação da linearidade da base apagada. Sendo assim, foi possível apresentar que o processo de redução vocabular na Libras não mostra preferência pelo apagamento de uma das bases especificamente, diferente do que ocorre no português brasileiro que tende a ter o morfema da base direita deletado (Vilela et al. 2007). Também foi percebido, devido à morfologia simultânea, que nem sempre é possível classificar a deletação em termos esquerdo ou direito, como é o caso do sinal para "juiz esportivo" que é um composto simultâneo. Isso pode ser visto como um reflexo do efeito de modalidade, ou seja, as línguas de sinais, por terem um caráter visuoespacial e, pelo menos, dois articuladores primários, as mãos, trazem possibilidades de articulação, diferente do que ocorre nas línguas orais.

Por fim, ressaltamos que, embora a redução vocabular não seja tão produtiva como os demais processos de formação de palavras, acreditamos que este processo merece atenção e novos estudos, uma vez que ele contribui para a expansão lexical e está intimamente ligado à economia linguística. Também acreditamos que este estudo abre possibilidades para futuras pesquisas e reflexões sobre o processo de recomposição nas línguas de sinais.

\section{Referências}

ARAÚJO, Gabriel. Truncamento e reduplicação no português brasileiro. Revista de Estudos da Linguagem. Belo Horizonte, v. 10, n. 1, p. 61-90, jan./jun. 2002.

ALVES, I. M. Neologismo: criação lexical. São Paulo: Ática, 1990.

BAGNO, Marcos. Gramática pedagógica do português brasileiro. São Paulo: Parábola Editorial, 2011.

CUNHA, C.; CINTRA, L. Nova gramática do português contemporâneo. 6. ed. Rio de Janeiro: Lexikon, 2013. 
A redução vocabular como processo de criação lexical: uma análise do fenômeno na LibrasLuiz Delmir Rildo Alves • Aline Garcia Rodero-Takahira

\section{DINIZ, Heloise Gripp. A História da Língua de Sinais Brasileira}

(Libras): um estudo descritivo de mudanças fonológicas e lexicais. 2010. 144 p. Dissertação (Mestrado em Linguística). Universidade Federal de Santa Catarina, Centro de Comunicação e Expressão, Programa de Pós-Graduação em Linguística, Florianópolis, 2010.

FARIA-NASCIMENTO, S. P.; CORREIA, M. Um olhar sobre a Morfologia dos gestos. Lisboa: Universidade Católica Editora, 2011.

FRISHBERG, N. Arbitrariness and Iconicity: Historical Change in American Sign Language. Language, v. 51, n. 3 (Sep., 1975), p. 696-719, 1975.

\section{GONÇALVES, Carlos Alexandre. Processos Morfológicos Não-}

Concatenativos no português do Brasil: tipos e funções. [versão revista e ampliada da Comunicação "Processos de redução vocabular: tipos e funções", apresentada na XVII Jornada de Estudos Lingüísticos do GELNE, que se realizou em Recife (instituto de Letras da UFPE) em setembro de 1999].

KLIMA, Edward S.; BELLUGI, Ursula. The Signs of Language. Cambridge, MA: Harvard University Press, 1979.

LIDDELL, Scott. K.; JOHNSON, Robert. American Sign Language compound formation processes, lexicalization, and phonological remnants. Natural Language and Linguistic Theory, v. 4, n. 8, p. 445-513, 1986.

MARTINET, A. Elementos de Lingüística Geral. Trad. J. Morais Barbosa, Lisboa: Sá da Costa, 1964.

OATES, Eugênio. Linguagem das mãos. Aparecida do Norte: Santuário, 1969.

OATES, Eugênio. Língua das Mãos. Adaptação e atualização e Simone Vecchio. Aparecida: Editora Santuário, 2017.

QUADROS, R. M.; KARNOPP, Lodenir. Língua de sinais brasileira: estudos lingüísticos. Porto Alegre: Artmed, 2004.

RODERO-TAKAHIRA, Aline Garcia. Compostos na língua de sinais brasileira. 2015. Tese (Doutorado em Semiótica e Lingüística Geral) Faculdade de Filosofia, Letras e Ciências Humanas, Universidade de São 
A redução vocabular como processo de criação lexical: uma análise do fenômeno na LibrasLuiz Delmir Rildo Alves • Aline Garcia Rodero-Takahira

Paulo, São Paulo, 2015. doi:10.11606/T.8.2015.tde-23112015-125742. Acesso em: 19 mar. 2018.

RODERO-TAKAHIRA, Aline Garcia; MEDEIROS, Davi Vieira. (Manuscrito). Formas de anotação da libras: em busca de uma proposta de padronização para uso acadêmico. Trabalho apresentado no "Il Simpósio do Grupo de Estudos em Língua de Sinais e Cognição (Lisco)", em agosto de 2016, na Universidade de São Paulo (USP).

RODERO-TAKAHIRA, Aline Garcia; SCHER, Ana Paula. (2020).

Classificando os Compostos da Libras. Porto Das Letras, 6(6), 152180. Recuperado de: https://sistemas.uft.edu.br/periodicos/index.php/ portodasletras/article/view/11439.

\section{SCHER, Ana Paula. A study of truncated nominal forms in Brazilian}

Portuguese. Their Derivation and their relation to nonverbal form classes. In: KATO, Mary Aizawa; ORDÓÑEZ, Francisco (Ed.). The Morphosyntax of Portuguese and Spanish in Latin America. Oxford studies in comparative syntax. New York, NY Oxford University Press. 2017. SCHER, Ana Paula. 2013. Concatenative affixation in Brazilian Portuguese Truncated forms. Proceedings of GLOW in Asia IX 2012. GOTO, Nobu; OTAKI, Koichi, SATO, Atsushi; TAKITA, Kensuke (Eds.). Disponível em: http://faculty.human.mie-u.ac.jp/ glow_mie/ IX_Proceedings_Oral/GLOWIXProceedings_Final.pdf. Acesso em: 19 mar. 2018.

STOKOE, William. Sign language structure: An outline of the visual communication systems of the American Deaf. Studies in Linguistics, Occasional Papers 8. Buffalo: University of Buffalo Press, 1960. VILELA, Ana Carolina. GODOY, Luisa. CRISTÓFARO-SILVA, Thais. Truncamento no Português Brasileiro: para uma melhor compreensão do fenômeno. UFMG. 2006. Disponível em: http://www.projetoaspa. org/cristofaro/publicacao/pdf/truncamento-rel- 2006ms.pdf. Acesso em: 19 mar. 2018. 